\title{
Ensaio de interação ou teste de usabilidade... afinal, do que estamos falando?
}

\author{
User trial ${ }^{1}$ or usability testing... after all, what are we talking about?
}

Stephania Padovani, André Schlemmer

ensaio de interação, teste de usabilidade, metodologia

O presente estudo teve como objetivo diferenciar dois métodos de envolvimento do usuário: ensaio de interação e teste de usabilidade. Para tanto, inicialmente, comparamos as duas expressões quanto aos termos utilizados, buscando seu significado e recorrendo a sinônimos. Em seguida, recorremos a referências na área de Design Centrado no Usuário que definissem e descrevessem as características de cada um dos métodos. Por fim, realizamos uma análise de 45 dissertações e teses brasileiras que aplicaram o ensaio de interação (de 1996 a 2020). Os resultados nos permitiram estabelecer diferenças entre o ensaio de interação e o teste de usabilidade no que tange aos objetivos, combinação de dimensões e métricas, tipo de tarefa realizada pelos participantes, nível de formalidade, combinação de técnicas de coleta e ênfase (qualitativa | quantitativa | híbrida) na coleta e análise dos dados.

user trial, usability testing, methodology

This study aimed to compare and contrast two user-involvement methods: user trials and usability testing. In order to achieve such, initially, we compared both expressions considering terms' meaning and synonyms. After that, we called upon User Centred Design references that defined and described the characteristics of each method. Lastly, we analysed 45 Brazilian thesis and dissertations that included user trials within their method (from 1996 to 2020). Results allowed us to establish a difference between user trials and usability testing in terms of objectives, dimensions and metrics, type of task carried out by participants, formality, combination of data-gathering techniques and emphasys (qualitative | quantitative | hybrid) on data collection and analysis.

\section{Introdução}

Envolver usuários no processo de projeto ou de pesquisa é uma atitude preconizada pelas abordagens de Design Centrado no Usuário (DCU), Design Centrado no Humano (DCH), Design Participativo (DP) e, mais recentemente, Design Thinking (DT). As formas de envolvimento variam amplamente em termos de frequência no processo de design e/ou intensidade de contribuição, mas também em relação aos métodos e técnicas utilizados para promover a participação do usuário.

\footnotetext{
${ }^{1}$ Não encontramos no idioma inglês uma tradução exata para o termo "ensaio de interação". Consideramos que "user trial" é a que mais se aproxima.
}

Anais do $10^{\circ} \mathrm{CIDI}$ e $10^{\circ} \mathrm{CONGIC}$

Kelli C.A.S. Smythe, Rafael de Castro Andrade (orgs.)

Sociedade Brasileira de Design da Informação - SBDI

Curitiba | Brasil | 2021
Proceedings of the $10^{\text {th }} \mathrm{CIDI}$ and $10^{\text {th }}$ CONGIC

Kelli C.A.S. Smythe, Rafael de Castro Andrade (orgs.)

Sociedade Brasileira de Design da Informação - SBDI Curitiba | Brazil | 2021 
No presente artigo, enfocamos especificamente o método de ensaio de interação, adotado com bastante frequência em pesquisas na área de Design da Informação, usualmente após a condução de estudo analítico (e.g., Castro Júnior, 2016; Napo, 2015; Hammerschmidt, 2014).

Em nossa revisão de literatura, a citação mais recorrente para explicar no que consiste um ensaio de interação cabe a Cybis (2003) em apostila do Laboratório de Utilizabilidade da UFSC. Curiosamente, em livro publicado posteriormente (Cybis et al., 2007 - $1^{\text {a }}$ edição; 2010 $2^{\mathrm{a}}$ edição), com conteúdo semelhante à apostila, os autores "abandonam" o termo "ensaio de interação", elencando apenas "observação do usuário" e "teste de usabilidade" como técnicas que guardam alguma semelhança com o ensaio de interação. Mais recentemente, entretanto, em nova edição do livro (Cybis et al., 2015) os autores passam a incluir o termo "ensaio de interface" dentre as técnicas de teste de interfaces com usuários.

Independente da lacuna temporal (2003 a 2015) na literatura, no que tange a referências que fundamentem/instrumentalizem a realização de ensaios de interação, o método e o termo continuaram sendo utilizados pela comunidade acadêmica de Design e áreas afins no Brasil. Para exemplificar tal constatação, Francisco e Benitti (2014) realizaram um mapeamento sistemático de pesquisas em usabilidade no Brasil e verificaram que o ensaio de interação foi o segundo método mais utilizado pelos pesquisadores, atrás apenas da técnica de questionário.

Refletindo sobre o contexto delineado, chegamos ao seguinte questionamento: visto que o termo "ensaio de interação" persistiu nas publicações, mesmo "ancorado" em referência pouco recente (2003) e de menor credibilidade acadêmica (apostila), no que exatamente um ensaio de interação se diferencia do teste de usabilidade, para justificar a adoção do primeiro termo?

Assim, como base nesse questionamento, o presente artigo apresenta, inicialmente, uma comparação conceitual / terminológica entre os termos "ensaio de interação" e "teste de usabilidade". Em seguida, com base em levantamento / análise de dissertações e teses nacionais que aplicaram o ensaio de interação, buscamos características metodológicas que nos permitam estabelecer uma clara diferenciação entre os dois métodos ${ }^{2}$.

\section{Fundamentação teórica}

\section{Teste de usabilidade}

De acordo com o Dicionário Aurélio (2010), a palavra teste significa "exame, verificação ou prova para determinar a qualidade, a natureza, ou o comportamento de alguma coisa ou de um sistema sob certas condições". Com base nessa definição, podemos afirmar que ao executarmos um teste de um sistema de informação, visamos verificar sua qualidade quando utilizado por um usuário humano.

Quando adicionamos "de usabilidade" à palavra teste, especificamos que aspecto da qualidade desse sistema será aferido. De modo a desambiguar o termo, recorremos à definição trazida pela ISO 9241 para o termo usabilidade: "capacidade de um sistema em permitir que

\footnotetext{
${ }^{2} \mathrm{O}$ termo "ensaio de interface" foi excluído de nossa pesquisa por não ter sido encontrado em dissertações / teses.
} 
usuários específicos atinjam metas específicas com eficácia, eficiência e satisfação em contextos específicos de uso".

Combinando as duas definições é possível concluir então que um teste de usabilidade visa avaliar a qualidade da interação entre um ser humano e um sistema em termos de eficácia, eficiência e satisfação desse usuário, sendo esses três aspectos necessariamente avaliados para que dada testagem seja intitulada um teste de usabilidade.

Além dessa reflexão terminológica inicial, cabe analisar referências específicas da área de DCU e verificar como alguns autores-chave definem e descrevem as condições de realização de testes de usabilidade.

Maguire (2001), por exemplo, em artigo clássico da área de DCU, utiliza o termo avaliação de usabilidade de forma mais generalista para englobar testes envolvendo usuários com diferentes níveis de formalidade: controlados (maior formalidade, sem interferência do pesquisador), assistidos (formalidade média, uso de verbalização e perguntas) e participativos (baixa formalidade, conversa durante a interação). O teste controlado, segundo o autor, seria recomendado para verificar se os requisitos de usabilidade (eficácia, eficiência e satisfação) foram atingidos. Esse teste envolve o uso de um protótipo de alta fidelidade, ambiente de testagem que se assemelhe ao mundo real e apenas a assistência que o usuário teria em situação real de uso (e.g., manual de uso, ajuda).

Para Rubin e Chisnell (2011), o teste de usabilidade é uma ferramenta de pesquisa que se utiliza de técnicas para coletar dados empíricos durante a observação de usuários finais representativos utilizando um produto para realizar tarefas reais. Segundo os autores, o teste deve ser conduzido no ambiente real de trabalho (ou em local que o represente) e se faz necessário coletar medidas qualitativas e quantitativas. Rubin e Chisnell elencam duas possíveis abordagens para o teste de usabilidade:

- teste formal (experimental e com vistas a confirmar ou refutar hipóteses);

- teste menos formal (ciclo iterativo para expor problemas e moldar progressivamente o produto).

Cybis et al. (2015), por sua vez, enquadram o teste de usabilidade dentro da categoria teste com o usuário. Os testes com usuários envolvem: "usuários reais ou representativos da população-alvo do sistema interagindo com o sistema para realizar tarefas específicas em um contexto de operação real ou simulado" (CYBIS et.al., 2015. p. 257). Para os autores, o teste de usabilidade se caracteriza como uma versão formal de teste com usuário, cujo interesse está nas medidas quantitativas de desempenho e da experiência do usuário.

Moran (2019), Especialista Sênior de User Experience do Nielsen Norman Group, explica que, em um teste de usabilidade, um pesquisador solicita a um participante que realize tarefas utilizando uma ou mais interfaces específicas. Enquanto o participante completa cada tarefa, o pesquisador observa seu comportamento e escuta seu feedback. A autora classifica os testes de usabilidade em dois tipos: 
- Qualitativo (foco na coleta de insights, descobertas e narrativas sobre como as pessoas utilizam produto ou serviço);

- Quantitativo (coleta de métricas para descrever a experiência do usuário; métricas mais comuns são completar a tarefa e tempo para realizar a tarefa).

Já Barnun (2021) define teste de usabilidade como o processo de aprender sobre os usuários, a partir dos próprios usuários, ao observá-los utilizando determinado produto para executar tarefas reais e atingir metas específicas que sejam de seu interesse. A autora divide os testes de usabilidade em dois tipos:

- teste formativo (produto em desenvolvimento, objetivo de diagnosticar e corrigir problemas, menor número de usuários, repetição durante processo de design);

- teste somativo (produto finalizado ou em fase de finalização, validação com base nos requisitos, maior número de usuários e realização de tratamento estatístico).

Por fim, de modo a incorporar também uma perspectiva mercadológica, cumpre citar o CIF (Common Industry Format), iniciativa do NIST (National Institute of Standards and Technology USA), criado para proporcionar maior consistência na descrição da realização dos testes de usabilidade (processo e resultados) em empresas / organizações. O documento se tornou uma norma ANSI em 2001 (ANSI/NCITS 354-2001), norma ISO em 2006 (ISO/IEC 25062:2006) e, em 2010, um documento customizável do NIST (NISTIR 7742) para EHR (Electronic Health Records). O CIF determina que todo teste de usabilidade deve envolver:

- participantes que sejam representativos em relação à população-alvo;

- tarefas representativas;

- métricas de eficácia (e.g., completar tarefas, erros), eficiência (e.g., tempo, desvios do caminho ótimo) e satisfação (e.g., ausência de desconforto e atitudes positivas em relação ao uso do produto).

Com base nas referências anteriormente citadas, torna-se possivel chegar a alguns pontos que caracterizam um teste de usabilidade. Tais características são representadas na figura 1, considerando o objetivo do teste, a situação de aplicação, os critérios de avaliação, as técnicas de coleta e as variações possíveis (quanto ao nível de formalidade, ênfase na coleta e estágio de desenvolvimento do produto a ser testado). 
Figura 1: Síntese das características de um teste de usabilidade

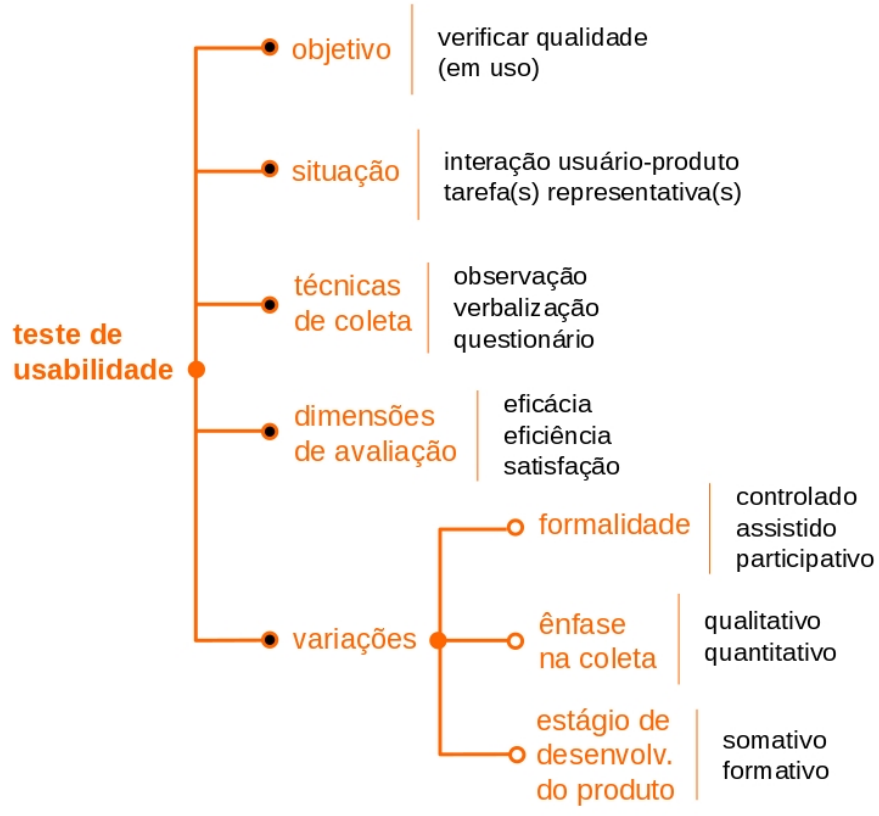

\section{Ensaio de interação}

Segundo o Dicionário Aurélio (2010), a palavra ensaio significa "prova ou experiência com o fim de verificar o desempenho de alguma coisa", tendo como sinônimos os termos análise, exame e apreciação. Já o termo desempenho apresenta como sinônimos (na mesma fonte), os termos atuação e comportamento. A partir dessa definição, podemos então afirmar que ao realizarmos um ensaio com um sistema de informação, visamos verificar como este desempenha suas funções ou como um usuário desempenha suas tarefas ao utilizar tal sistema.

Se acrescentarmos à palavra ensaio o termo interação (definido por Hornbaek e Oulasvirta (2017) como o processo que ocorre quando duas entidades determinam mutuamente seu comportamento), passamos a considerar que o desempenho que se deseja verificar se refere à relação / processo de comunicação estabelecido entre o usuário e o sistema. Diferentemente do termo usabilidade, o termo interação não traz em sua definição a especificação de dimensões a serem avaliadas.

Após essa reflexão terminológica, cumpre citar autores da área de DCU que explicitem as características do método de ensaio de interação. Na referência mais antiga que encontramos, Moço (1996) descreve o ensaio de interação como consistindo de uma observação do trabalho real com um aplicativo em uma situação realista. Durante a aplicação desse método, o analista busca possíveis problemas que a interação causa ao usuário e à organização de seu trabalho. A autora explica que, por intermédio dos ensaios de interação, os analistas podem compreender como o usuário elabora e executa sua tarefa com o auxílio do software, validar (ou não) hipóteses de mal funcionamento de funções ou comprovar a utilidade de um software em relação às necessidades do usuário.

Para Cybis (2003), um ensaio de interação consiste de uma simulação de uso do sistema da qual participam pessoas representativas do público-alvo. O autor explica que, durante o uso, 
os participantes tentam fazer tarefas típicas de suas atividades, com uma versão do sistema pretendido. Quanto ao local de realização, Cybis aponta que os ensaios podem ser realizados tanto em laboratório, quanto no próprio local de trabalho dos usuários.

Segundo Betiol (2004), os ensaios de interação são uma simulação de uso do sistema. A autora explica que, nos ensaios de interação, usuários representativos executam tarefas determinadas com uma versão do produto, podendo esta variar desde um protótipo em papel até a sua versão final. Quanto ao local de realização, Betiol explica que os ensaios de interação podem ser conduzidos tanto em laboratório (fixo ou portátil) quanto no próprio local em que os usuários realizam suas tarefas (ambiente real de uso). Além da observação, a autora recomenda o uso da técnica de verbalização (simultânea ou consecutiva) caso se deseje saber porque o usuário realiza determinadas ações.

Conforme Dias (2007), o ensaio de interação faz parte da categoria de avaliação intitulada testes empíricos de usabilidade. Segundo a autora, esse método busca coletar dados qualitativos ou quantitativos por intermédio da observação da interação usuário-sistema. Os participantes precisam ser usuários representativos do sistema e devem realizar tarefas reais. Ainda segundo Dias, o ensaio de interação prioriza aferir a qualidade das interações que se estabelecem entre o usuário e o sistema. Para tanto, além da observação, podem ser utilizadas as técnicas de verbalização ou co-descoberta.

Por fim, retornamos a Cybis, em publicação mais recente (Cybis et al., 2015) na qual os autores, agora adotando o termo "ensaios de interface", explicam que esse método possui um nível de formalidade menor do que os testes de usabilidade, aproximando-se de uma avaliação participativa, com o interesse mais voltado aos aspectos qualitativos das opiniões dos usuários e das estratégias de uso / superação de problemas. Ainda segundo os autores, os ensaios de interface são realizados usualmente no próprio local de trabalho do usuário.

Com base nas referências anteriormente citadas, torna-se possível chegar a alguns pontos que caracterizam um ensaio de interação. Tais características aparecem representadas na figura 2, considerando-se os mesmos parâmetros utilizados para caracterizar o teste de usabilidade (figura 1).

Ao compararmos as duas figuras, torna-se possível identificar algumas diferenças entre o teste de usabilidade e o ensaio de interação. O ensaio de interação possui maior pluralidade de objetivos, não possui dimensões de avaliação fixas, seu nível de formalidade enquadra-se entre o assistivo e o participativo e foi citado pela maioria dos autores como de natureza qualitativa. Na segunda parte do presente artigo, visamos verificar as diferenças do ensaio de interação para o teste de usabilidade na prática, ou seja, na forma como o ensaio vêm sendo aplicado em teses e dissertações nacionais. 
Figura 2: Síntese das características de um ensaio de interação

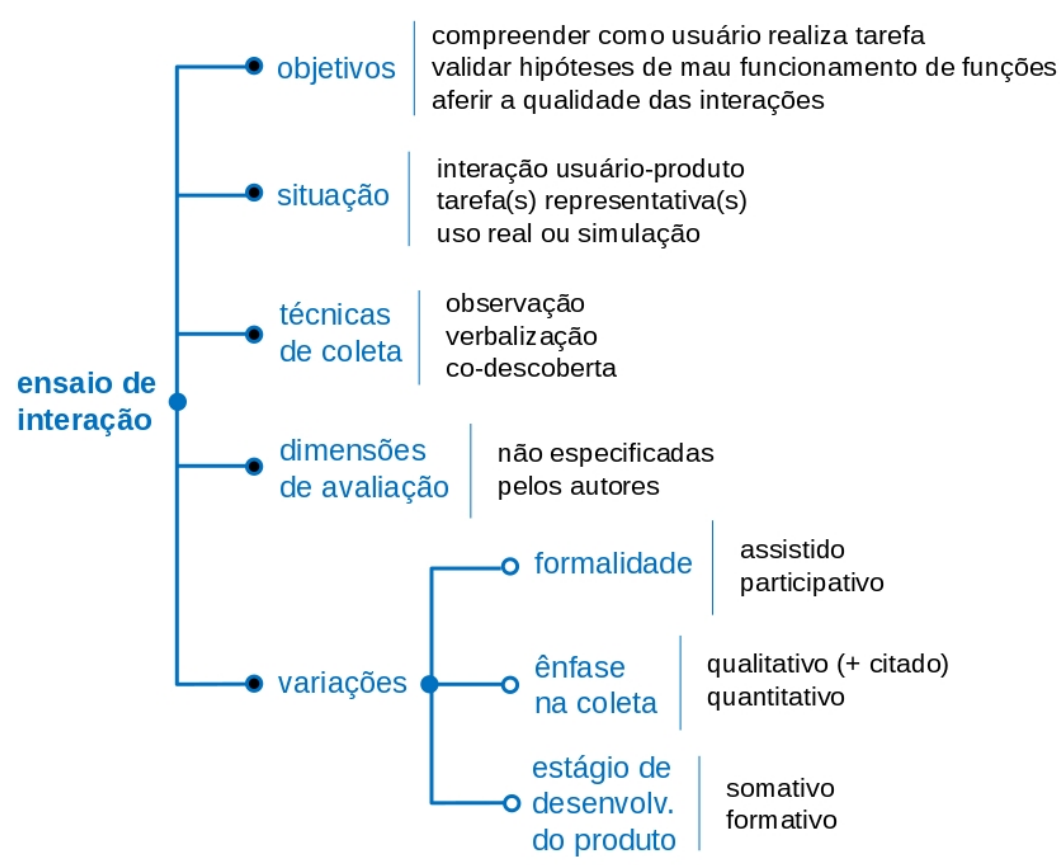

\section{Método}

No presente estudo, realizamos uma análise de teses e dissertações de IES brasileiras que aplicaram o ensaio de interação. A opção por analisar teses e dissertações (e não artigos científicos) se deve ao fato daqueles documentos possuírem maior detalhamento metodológico. Para tanto, buscamos pelo termo "ensaio de interação" nas seguintes bases:

- Catálogo de teses e dissertações da CAPES;

- Biblioteca Digital Brasileira de Teses e Dissertações;

- Repositórios de Teses e Dissertações das IES que retornaram resultados na busca às duas primeiras bases (UFAL, UFAM, UFMA, UFMG, UFPB, UFPR, UFRN, UFSC, UFU, UnB, UNESP).

Após essa primeira busca, filtramos os documentos encontrados para eliminar as teses e dissertações que lidavam com ensaios clínicos (e.g., área de Bioquímica) de interação e, em seguida, verificamos se a tese / dissertação havia aplicado o ensaio de interação ou apenas mencionado a expressão em alguma parte do trabalho. Apenas os trabalhos que aplicaram o método foram mantidos, o que nos gerou um total de 45 documentos para análise.

Para cada um dos trabalhos analisados, consideramos:

- nível na pós-graduação, ano de publicação e área do conhecimento;

- nomenclatura utilizada para se referir ao ensaio de interação;

- objetivo de aplicação do ensaio;

- dimensões e métricas; 
- tipo de tarefa (rígida | flexível);

- técnicas de coleta;

- nível de formalidade (controlado, assistido, participativo);

- ênfase na coleta | análise de dados (qualitativa, quantitativa, híbrida).

\section{Resultados e discussão}

Inicialmente, de modo a caracterizar a amostra analisada, apresentamos a distribuição dos documentos quanto ao ano de publicação, área do conhecimento e nível na pós-graduação. Verificamos que os documentos foram publicados entre 1996 e 2020, com maior incidência em 2013 e 2016. Do total de 45 documentos analisados, 33 são dissertações de mestrado e 12 teses de doutorado. Quanto à área do conhecimento, destacam-se Design, Engenharia de Produção, Ciência da Computação e Ciência da Informação.

Figura 3: Teses e dissertações que aplicaram ensaio de interação (por ano de publicação)

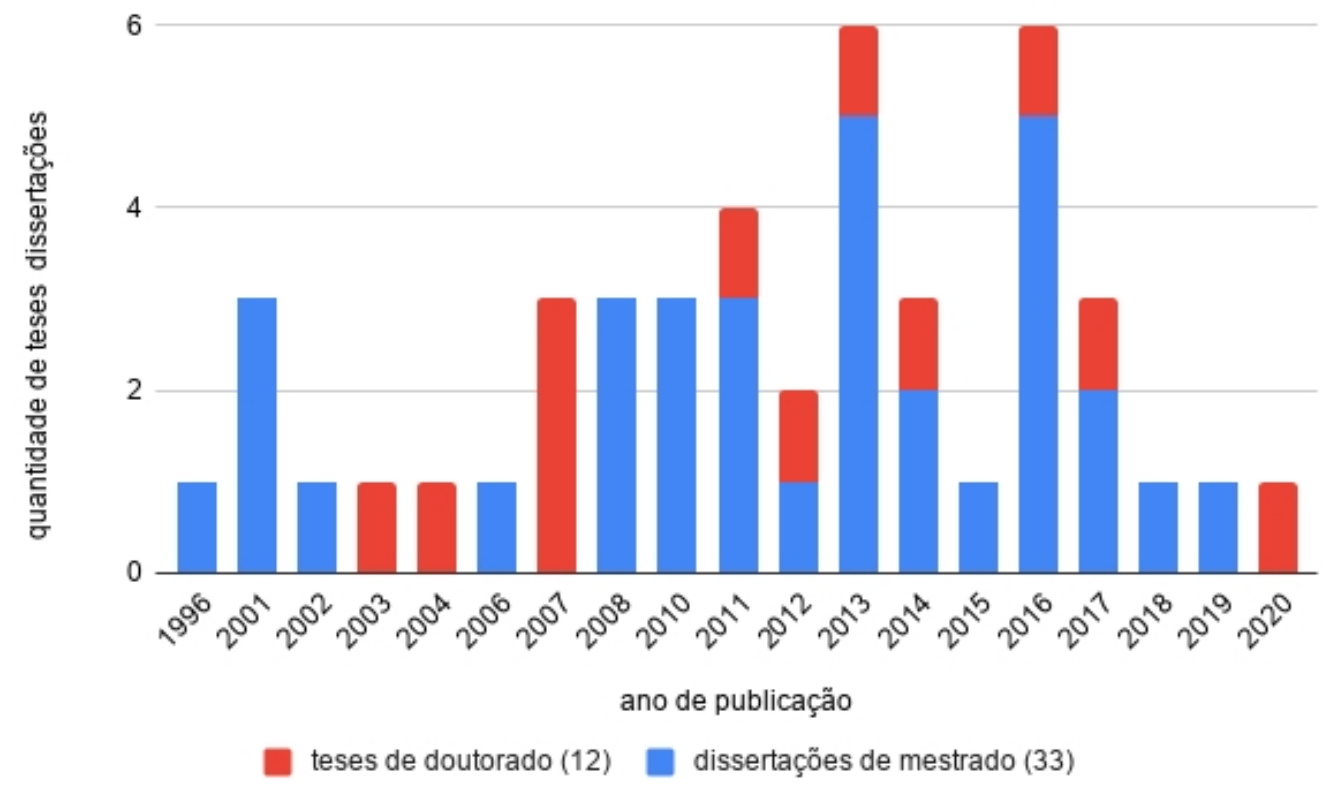

Figura 4: Teses e dissertações que aplicaram ensaio de interação (por área do conhecimento)

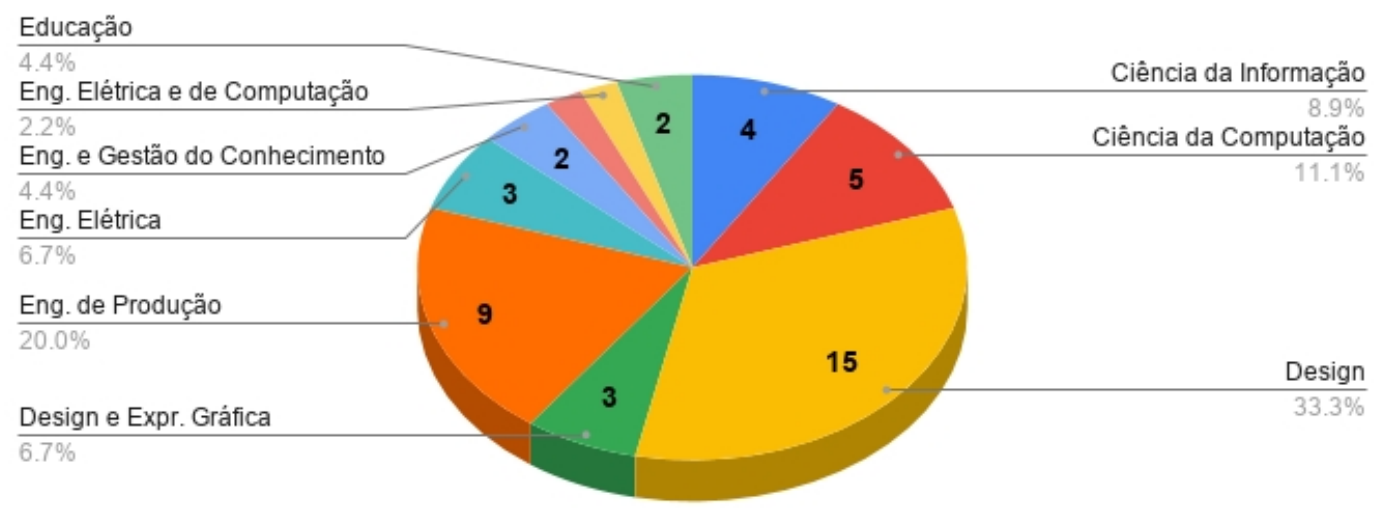


Após elencar algumas características gerais dos documentos analisados, considerando que um dos enfoques do presente artigo recai sobre a precisão da nomenclatura, cumpre identificar os termos utilizados para se referir aos ensaios de interação. Conforme representado na figura 5 , a maioria dos trabalhos $(73.3 \%)$ utilizou apenas o termo ensaio de interação para se referir à pesquisa conduzida com os usuários, enquanto o restante dos trabalhos utilizou até 3 termos diferentes. Na figura 6 , apresentamos as combinações de termos utilizados nas teses $\mathrm{e}$ dissertações que não se ativeram ao termo ensaio de interação e utilizaram, como sinônimos deste, termos que não possuem o mesmo sentido.

Figura 5: Quantidade de termos utilizados nos documentos para se referir aos ensaios de interação

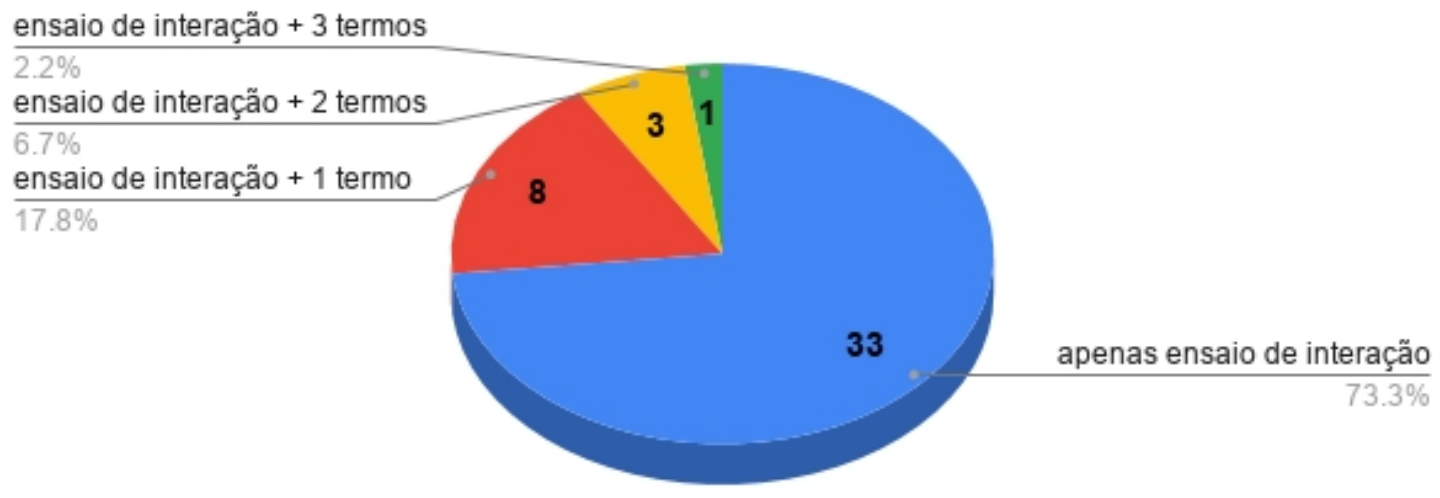

Figura 6: Termos utilizados para se referir ao ensaio de interação

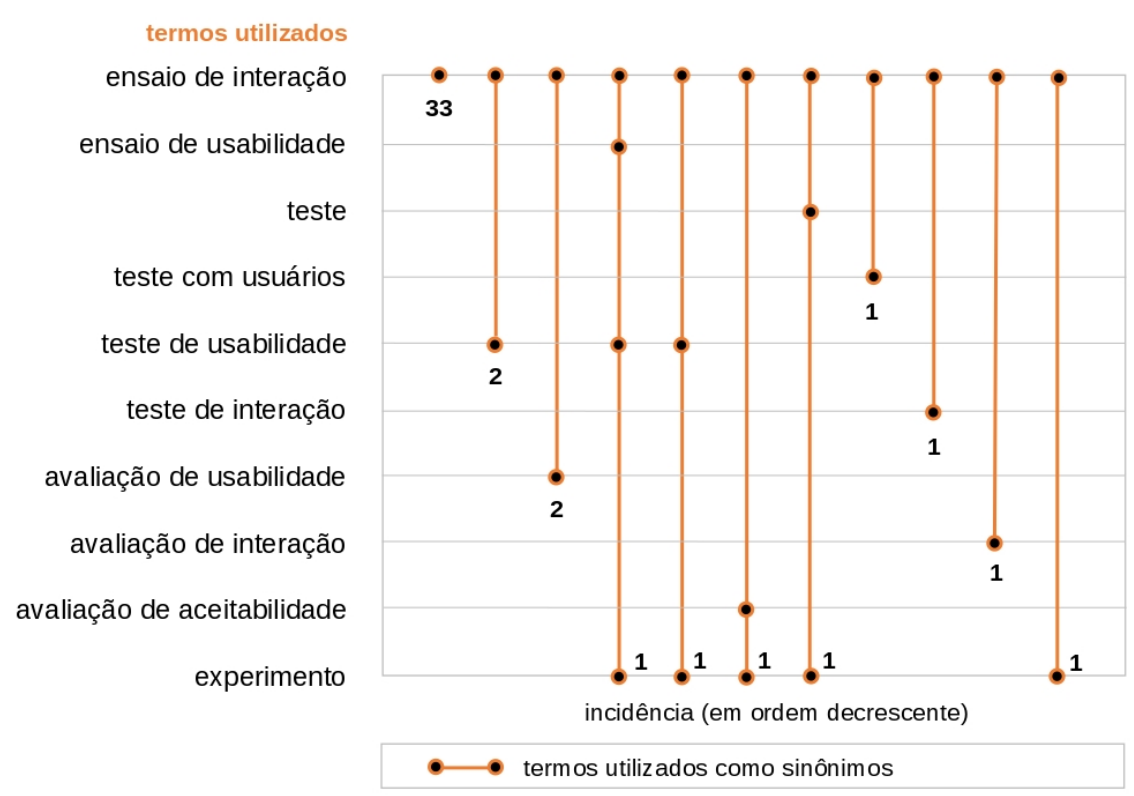

No que tange aos objetivos de aplicação do ensaio de interação (figura 7 ), apenas $35.6 \%$ apresentaram como propósito do ensaio "avaliar", mesma proporção atingida por "verificar". Com menor frequência, encontramos os propósitos de "identificar" (11.1\%), "comparar" (6.7\%) e "compreender" (6.7\%). Nesse sentido, a maioria dos trabalhos aplicou o ensaio de interação 
com objetivo diferente do teste de usabilidade, cujo objetivo é avaliar a capacidade do sistema em permitir que os usuários atinjam suas metas com eficácia, eficiência e satisfação.

Figura 7: Objetivos de aplicação dos ensaios de interação

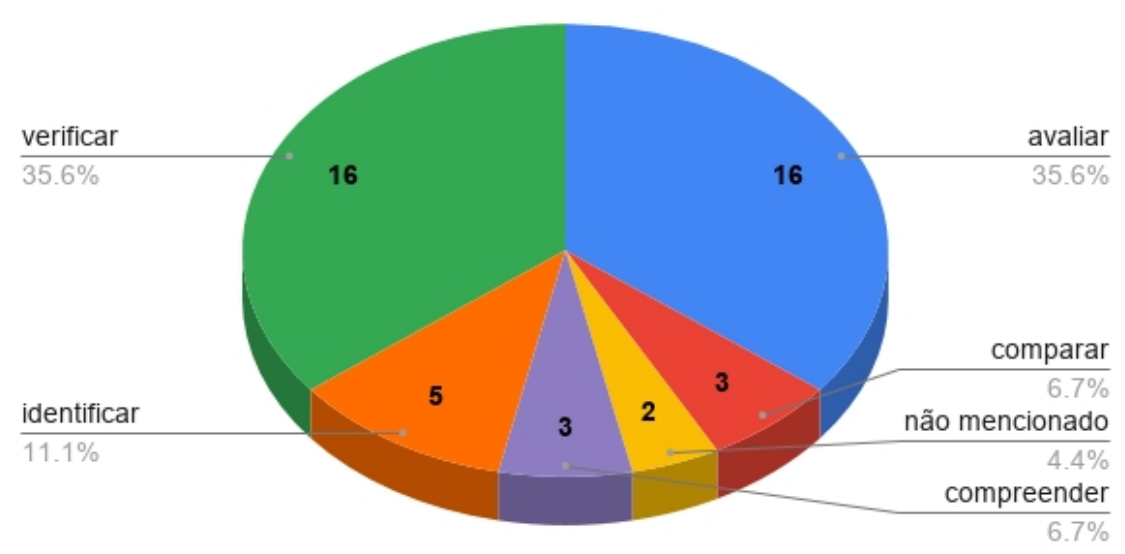

De modo a seguir contrastando ensaio de interação e teste de usabilidade, expandimos as categorias onde encontramos objetivos semelhantes ao teste de usabilidade (avaliar, verificar e identificar) nas figuras 8,9 e 10 .

Figura 8: Objetivos dos ensaios de interação (categoria avaliar)

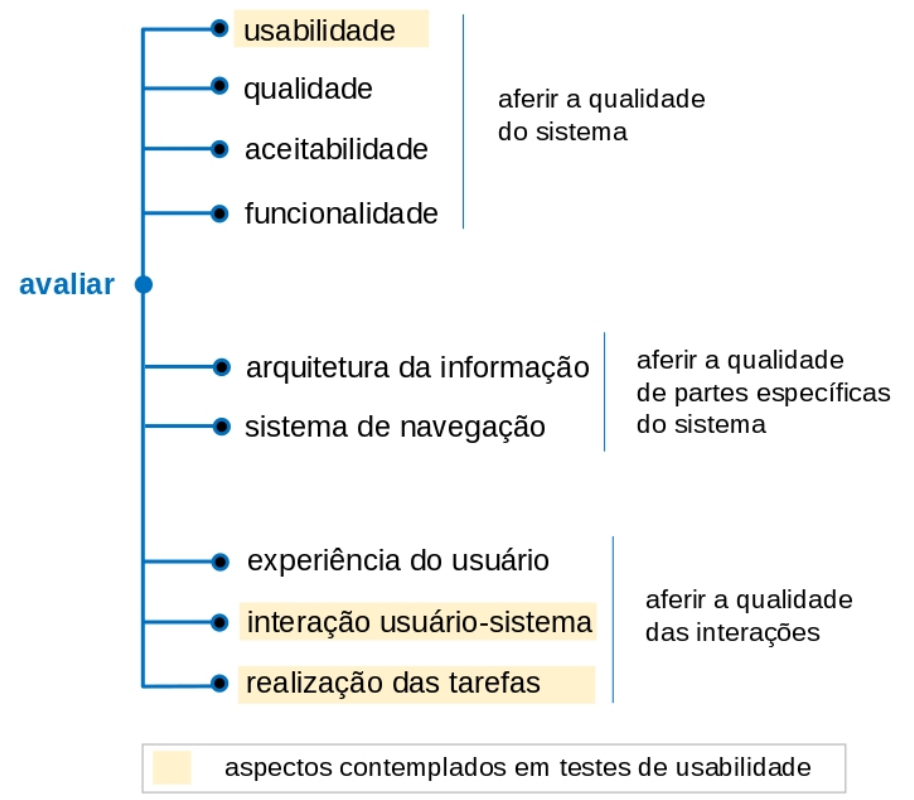

No que se refere aos objetivos dentro da categoria "avaliar", encontramos apenas dois documentos que mencionaram "avaliar usabilidade (...)". Os dois utilizaram como dimensões de avaliação eficácia, eficiência e satisfação, caracterizando-se, portanto, como testes de usabilidade, apesar de terem utilizado o termo ensaio de interação. Já os objetivos "avaliar a interação usuário-sistema" e "a realização de tarefas" também podem ocorrer em testes de 
usabilidade, mas nos casos dos documentos analisados, essa avaliação não envolveu a combinação de eficácia, eficiência e satisfação.

Figura 9: Objetivos dos ensaios de interação (categoria verificar)

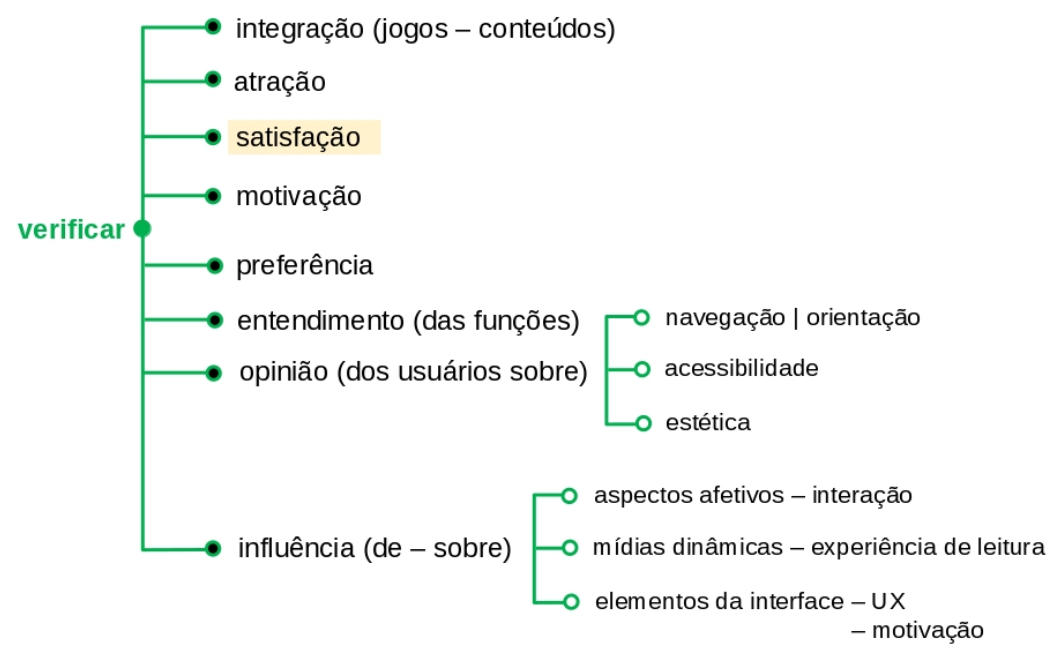

aspectos contemplados em testes de usabilidade

Dentro da categoria "verificar", encontramos apenas "satisfação" como sendo uma dimensão coincidente com o teste de usabilidade. Todas os outros complementos ao verbo verificar não caracterizam testes de usabilidade.

Figura 10: Objetivos dos ensaios de interação (categoria identificar)

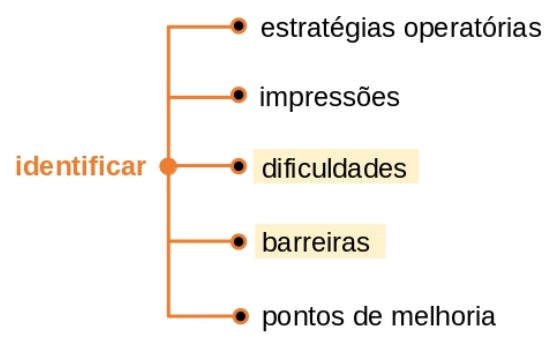

aspectos contemplados em testes de usabilidade

Por fim, dentro da categoria "identificar", encontramos "dificuldades" e "barreiras" como sendo aspectos coincidentes com o teste de usabilidade, mas não exclusivos desse tipo de avaliação. Os outros aspectos mencionados não caracterizam testes de usabilidade.

Além de analisar os objetivos, identificamos as dimensões e métricas associadas aos ensaios de interação nas teses e dissertações. Verificamos (vide figura 11) que em apenas $29.7 \%$ dos trabalhos houve coleta de dados de natureza objetiva e subjetiva, como é característico dos testes de usabilidade. Em contraste, em $45.9 \%$ dos trabalhos, coletaram-se apenas dados subjetivos e nos $24.3 \%$ restantes apenas dados objetivos. 
Figura 11: Divisão das dimensões e métricas quanto a subjetividade | objetividade

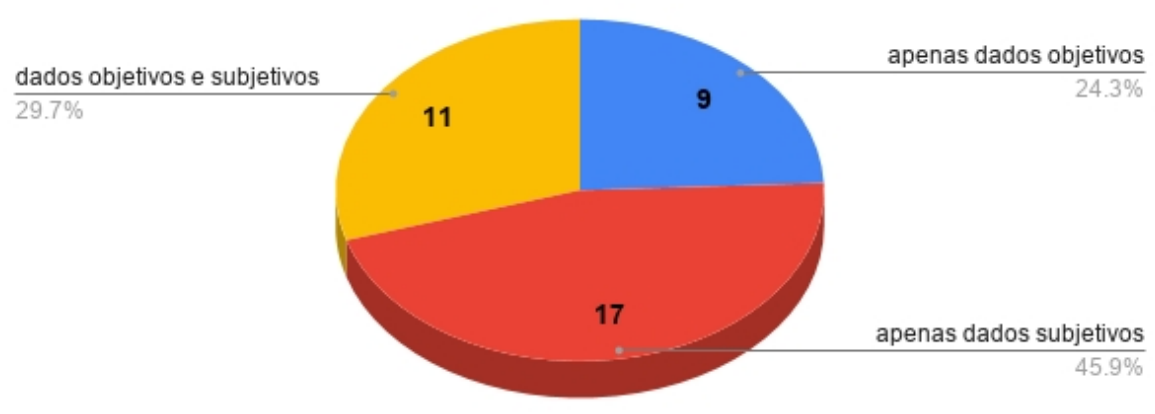

Ao analisarmos as dimensões e métricas objetivas mensuradas a partir dos ensaios de interação, observamos grande comunalidade com os testes de usabilidade. Conforme representado na figura 12, eficácia, eficiência, erros, tempo e caminho ótimo são todas passíveis de utilização também em testes de usabilidade.

Figura 12: Dimensões e métricas objetivas

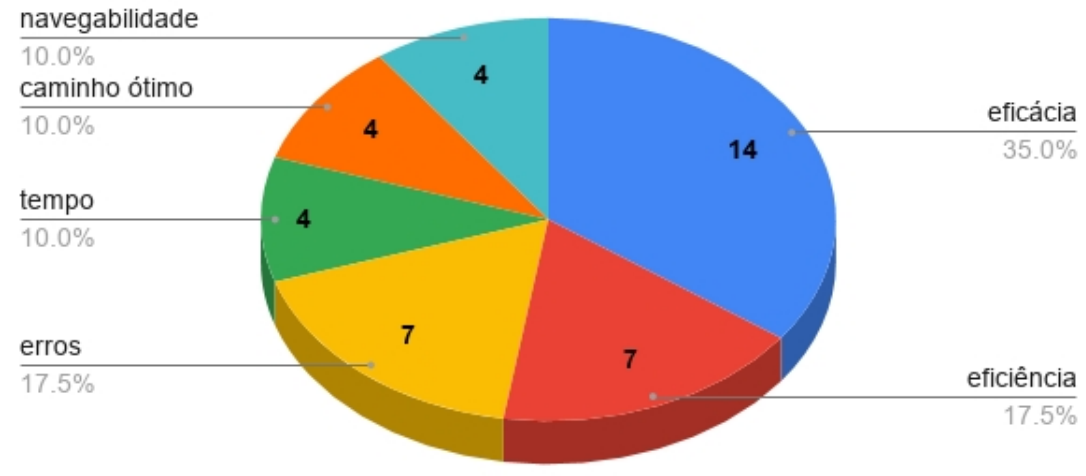

A situação se inverte quando analisamos as dimensões e métricas de natureza subjetiva. Conforme observamos na figura 13 , apenas $26.1 \%$ dos ensaios utilizaram como dimensão a satisfação, necessária na realização de testes de usabilidade. Dentre as demais, apenas a facilidade de uso (6.5\%) caberia, por exemplo, em um questionário de satisfação (debriefing).

Figura 13: Dimensões e métricas subjetivas

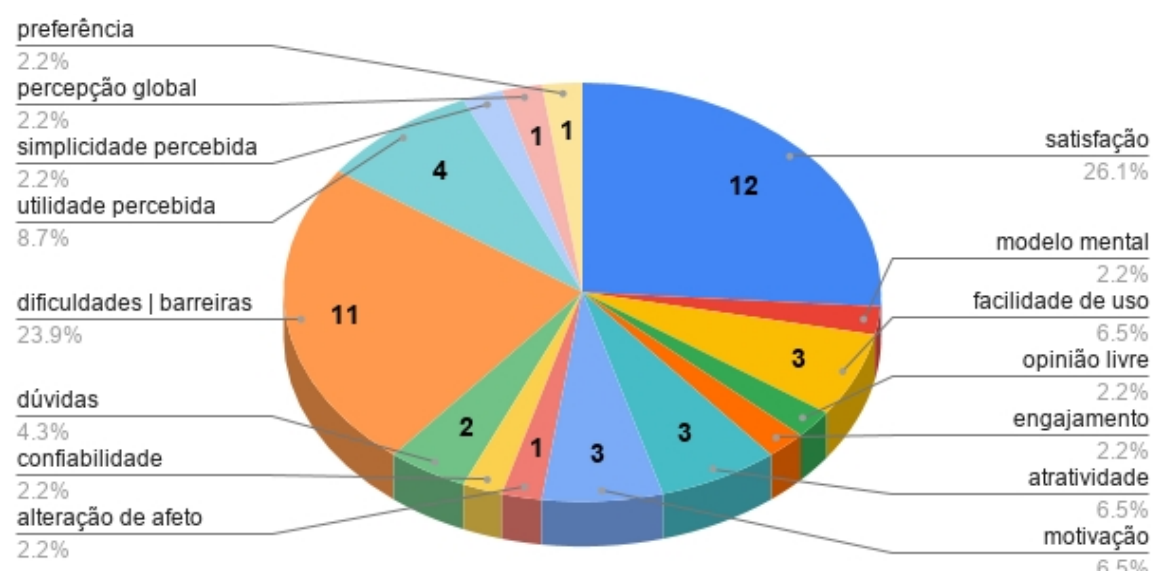


Devido às semelhanças encontradas nas dimensões e métricas utilizadas nos ensaios de interação em comparação com testes de usabilidade, selecionamos os trabalhos que utilizaram mais de uma dimensão de usabilidade (eficácia, eficiência e satisfação), para uma análise mais aprofundada. Conforme observamos na figura 14, 2 trabalhos (G1) utilizaram a combinação eficácia + eficiência + satisfação, caracterizando-se como testes de usabilidade, apesar de nomearem a pesquisa com usuários como ensaio de interação. Outros 2 trabalhos (G2) utilizaram essa combinação, mas acrescentaram outras dimensões, o que estaria além do escopo da usabilidade. Um terceiro grupo de trabalhos (G3) utilizou a combinação eficácia + eficiência, mas não incluiu a satisfação. Por fim, um quarto grupo de trabalhos utilizou a combinação eficácia + satisfação, mas não incluiu a eficiência. Ou seja, apesar dos ensaios de interação utilizarem dimensões semelhantes às do teste de usabilidade, apenas em 2 trabalhos (em um total de 45) a combinação foi exatamente a mesma (eficácia + eficiência + satisfação).

Figura 14: Combinações de dimensões e métricas utilizadas nos ensaios de interação que mais se aproximaram do teste de usabilidade

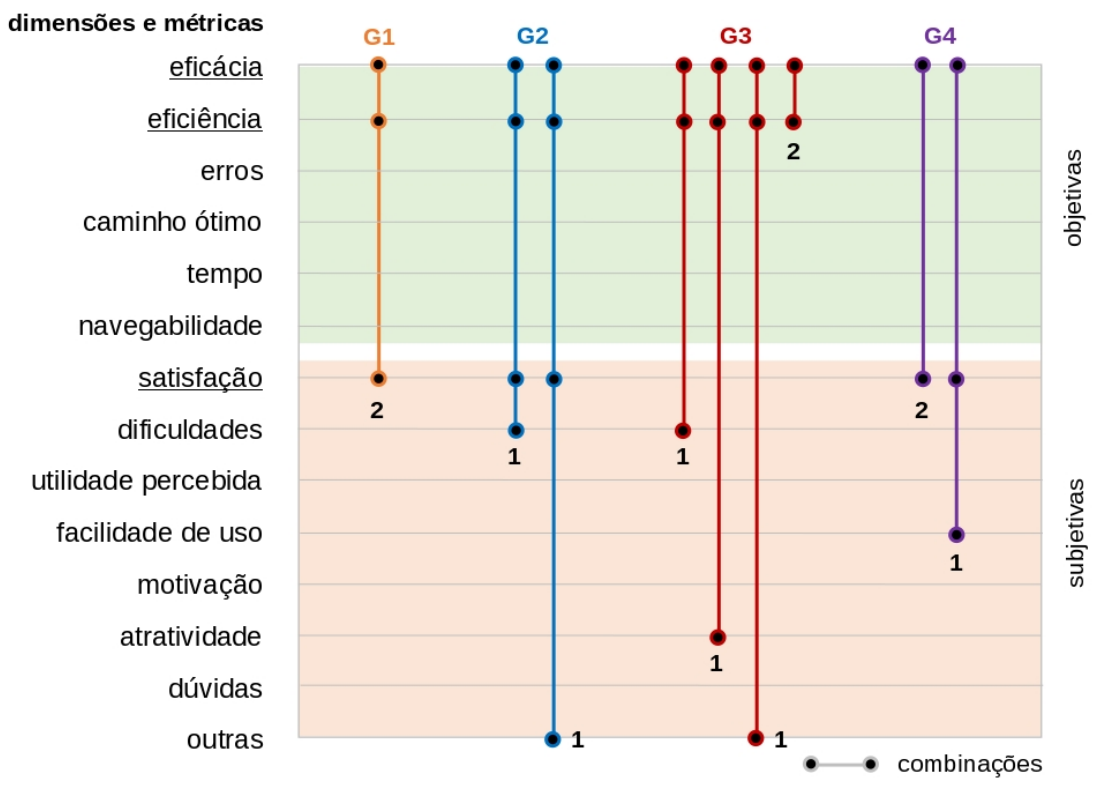

A seleção das dimensões e métricas está diretamente associada ao tipo de tarefa que os usuários realizarão no sistema. Nesse artigo utilizamos (com base em Bates, 2002) os termos:

- tarefa rígida (para tarefas de busca | ações específicas e que nos permitem afirmar se foram concluídas com sucesso, se ocorreram erros e traçar um caminho ótimo ${ }^{3}$ );

- tarefa flexível (para tarefas de navegação mais livre e com diversas possibilidades de caminhos | soluções pelos usuários).

\footnotetext{
${ }^{3} \mathrm{O}$ caminho mais curto, ou seja, mais eficiente, entre o ponto de início da tarefa e o ponto em que a tarefa foi concluída com sucesso.
} 
Entendemos que tarefas rígidas nos permitem mensurar, com precisão, eficácia e eficiência, ao contrário do que ocorre com as tarefas flexíveis. Nesse sentido, pressupomos que os ensaios de interação utilizariam em sua maioria tarefas flexíveis, o que se confirmou ao observarmos os resultados apresentados na figura 15.

Figura 15: Tipos de tarefas realizadas pelos participantes dos ensaios de interação

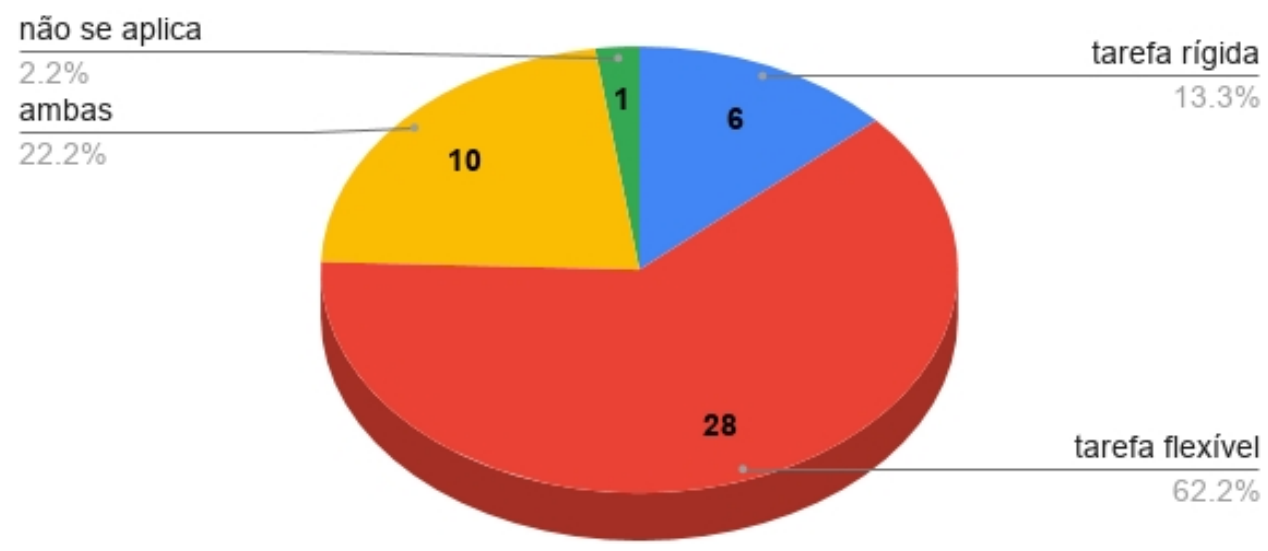

No que se refere às técnicas de coleta nos ensaios (figura 16), verificamos que as mais utilizadas foram a observação (35.6\%), seguida do questionário (25.6\%), ambas necessárias à composição tradicional dos testes de usabilidade. Entretanto, nenhuma das duas atingiu a maioria, o que revela uma maior diversidade de técnicas aplicadas nos ensaios de interação.

Figura 16: Técnicas de coleta utilizadas nos ensaios de interação

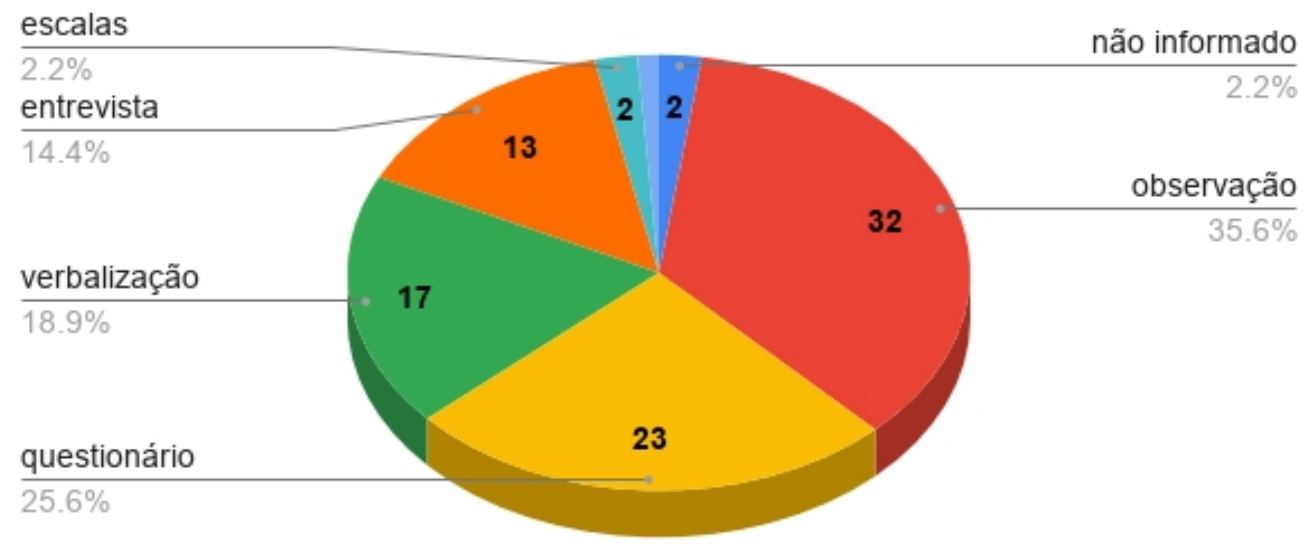

Ao analisarmos a combinação de técnicas empregadas (figura 17), observamos que apenas 11 trabalhos (em um total de 45) utilizaram a mesma combinação de técnicas do teste de usabilidade tradicional (observação + questionário). Ou seja, observação e questionário também foram adotados como técnica única ou combinados com outras técnicas, como por exemplo, verbalização, entrevistas e escalas. Nesse sentido, os ensaios de interação se diferenciaram dos testes de usabilidade pela multiplicidade de arranjos entre diferentes técnicas de coleta. 
Figura 17: Combinação de técnicas de coleta utilizadas nos ensaios de interação

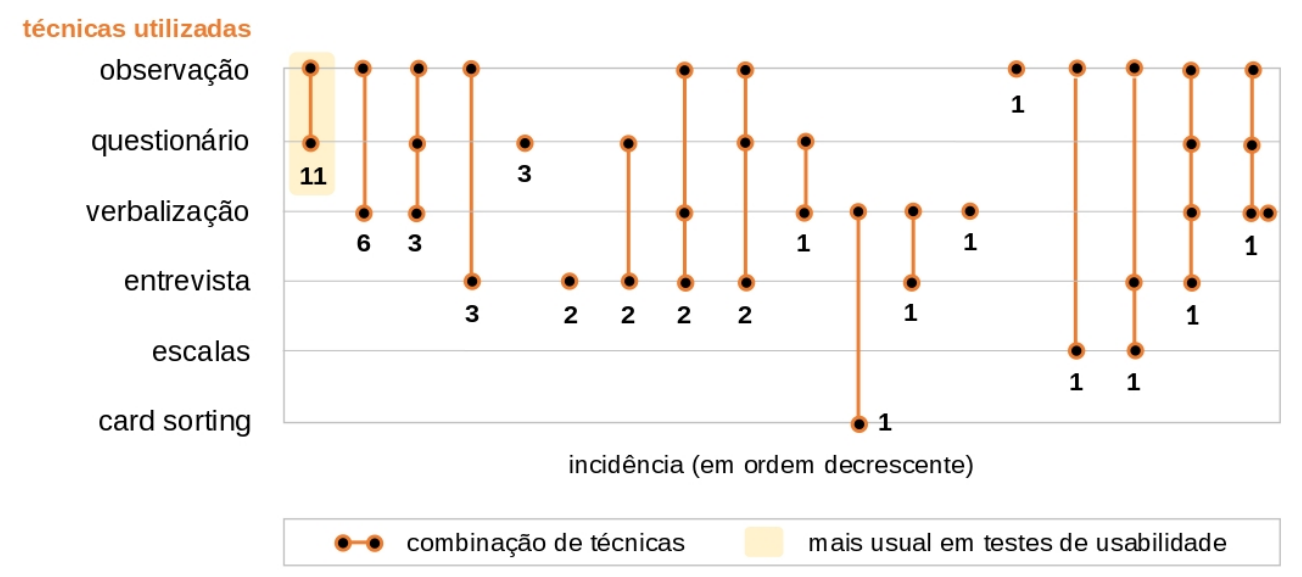

Outro aspecto que analisamos nos ensaios de interação foi o nível de formalidade adotado durante a condução dos mesmos. Os resultados demonstraram que a maioria dos ensaios adotou o modo assistido ou participativo (62.6\%). Esses dados confirmam a diferenciação pontuada pela literatura consultada que atribui menor formalidade aos ensaios de interação. Chamou-nos a atenção, entretanto, a frequência relativamente alta de ensaios realizados no modo controlado (37.5\%). Analisando pormenorizadamente esses estudos, verificamos que 2 se configuram como testes de usabilidade e os demais optaram por realizar interferência mínima durante a sessão de teste seja pelo perfil dos usuários (e.g., cegos utilizando leitor de tela) pela natureza do sistema (e.g., jogos - interferência do avaliador poderia prejudicar a imersão e o desenrolar da partida) ou para manter a baixa interferência externa em semelhança à realização da tarefa em seu ambiente real.

Figura 18: Nível de formalidade adotado durante a condução dos ensaios de interação

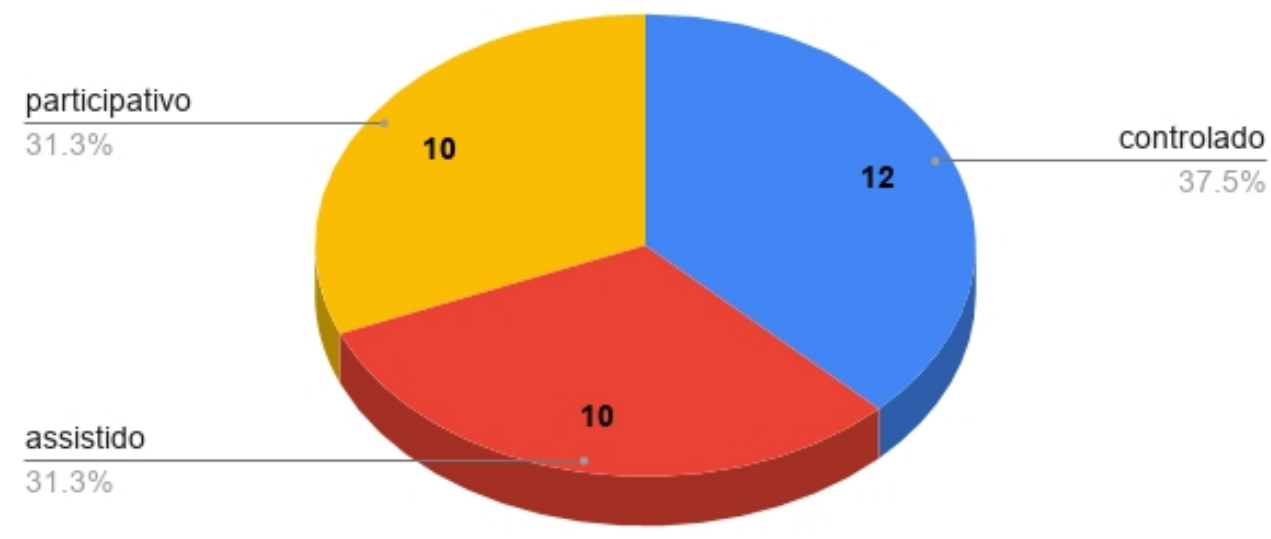

Por fim, analisamos os trabalhos quanto à ênfase na coleta e análise dos dados. Os resultados confirmaram, em acordo com o que a revisão de literatura apontou, que a maioria dos trabalhos utilizou uma abordagem qualitativa nos ensaios de interação (conforme representado na figura 19$)$. 
Figura 19: Ênfase na coleta | análise de dados

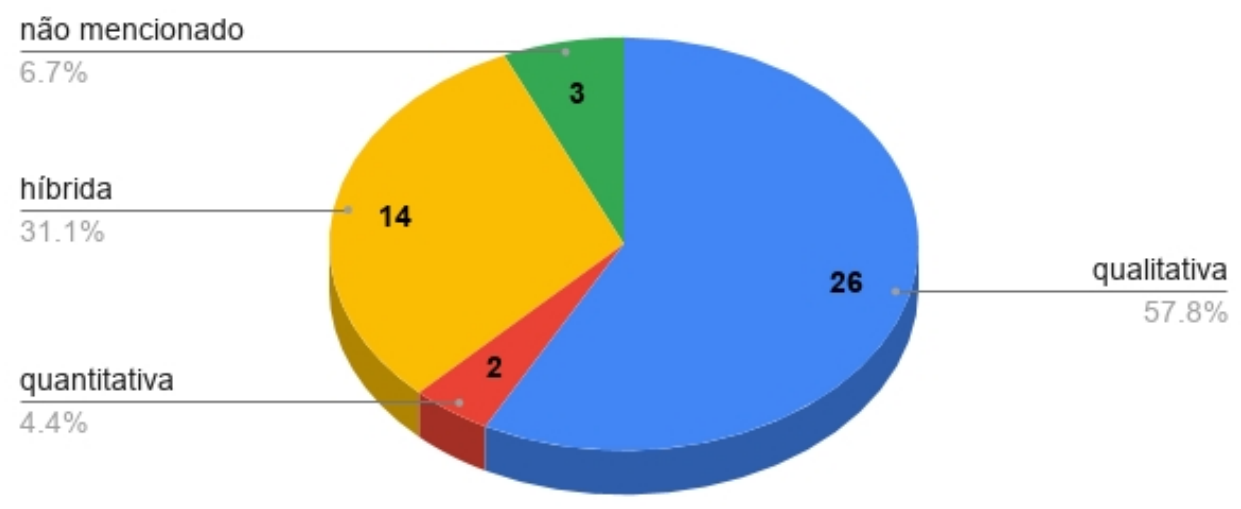

\section{Conclusões}

O presente estudo teve como objetivo diferenciar o método de ensaio de interação do teste de usabilidade. A comparação entre os resultados obtidos na revisão de literatura com aqueles oriundos da análise de teses e dissertações nacionais nos permitiu chegar a um conjunto de especificidades do ensaio de interação:

- objetivo: pode ser utilizado com o propósito de avaliar, mas também de compreender qualquer aspecto ou relação que ocorra durante a interação de um usuário com um sistema de informação;

- dimensões e métricas: pode utilizar apenas dimensões e métricas objetivas, apenas subjetivas ou combinação de ambas; as quais devem ser passíveis de mensuração enquanto ocorre a interação ou como consequência da interação; a combinação eficácia + eficiência + satisfação caracteriza o teste de usabilidade e, nesses casos, essa deve ser a nomenclatura utilizada;

- tipo de tarefa: adequado à investigação de tarefas rígidas, flexíveis ou combinação de ambas, mas verificamos maior frequência de realização de tarefas flexíveis;

- técnicas de coleta: pode ser conduzido utilizando qualquer técnica que permita conhecer os componentes operacional, cognitivo ou emocional da tarefa, combinados ou isoladamente, para tarefas individuais ou colaborativas; em nosso estudo as técnicas mais frequentes foram observação, entrevista, questionário e verbalização;

- nível de formalidade: predominam os modos assistido ou participativo, mas também pode ser conduzido no modo controlado se o perfil do usuário, natureza do sistema ou situação do ambiente assim demandar;

- ênfase na coleta | análise de dados: predominantemente qualitativa ou híbrida, por conta da maior flexibilidade e informalidade do método.

Ainda que tenhamos atingido nosso objetivo, cabe uma reflexão metodológica. Nosso trabalho foi, por vezes, bastante dificultado devido à ausência | incompletude de informações necessárias ao entendimento da aplicação dos ensaios de interação; mesmo em se tratando 
de teses e dissertações. Portanto, com vistas ao entendimento do leitor, à replicabilidade à maior confiabilidade dos resultados obtidos, consideramos que a descrição de ensaios de interação em teses e dissertações deve incluir informação sobre:

- objetivo específico do ensaio de interação;

- perfil dos participantes;

- ambiente de condução dos ensaios;

- tarefas a serem realizadas pelos participantes;

- dimensões e métricas;

- técnicas de coleta;

- nível de formalidade;

- estratégia de tratamento e análise de dados.

Por fim, quanto à forma de nomear o método, sugerimos que seja mantido "ensaio de interação" ao invés de "ensaio de interface" (Cybis et al., 2015), visto que o enfoque desse tipo de método está sobre a investigação do processo de uso de um sistema por seus usuários e não sobre e exame tão somente das características da interface. Acreditamos, ainda, que utilizar o termo "ensaio de interface" pode confundi-lo com métodos de inspeção de interface, conduzidos sem a participação de usuários.

\section{Referências}

Barnun, C. M. (2021). Usability testing essentials: ready, set... test! Burlington (MA): Morgan Kaufmann.

Bates, M. J. (2002). Toward an Integrated Model of Information Seeking and Searching. New Review of Information Behaviour Research, 3, 1-15.

Betiol, A. H. (2004). Avaliação de usabilidade para os computadores de mão: um estudo comparativo entre três abordagens para ensaios de interação. [Tese de doutorado]. Programa de Pós-graduação em Engenharia de Produção, Universidade Federal de Santa Catarina, Brasil.

Castro Júnior, E. F. (2016). A experiência do usuário (UX) em interfaces gráfico-informacionais: um estudo de caso dos aplicativos de educação em saúde da UNASUS / UFMA.

[Dissertação de mestrado]. Programa de Pós-graduação em Design, Universidade Federal do Maranhão, Brasil.

Cybis, W. A. (2003). Engenharia de usabilidade: uma abordagem ergonômica. Apostila do Laboratório de Utilizabilidade de Informática, Universidade Federal de Santa Catarina.

Cybis, W. A., Betiol, A. H., \& Faust, R. (2007). (2010). (2015). Ergonomia e usabilidade: conhecimentos, métodos e aplicações. São Paulo: Novatec Editora.

Ferreira, A. B. H. (2010). Dicionário Aurélio da Língua Portuguesa. Curitiba: Editora Positivo.

Dias, C. (2007). Usabilidade na web: criando portais mais acessíveis. Rio de Janeiro: Alta Books. 
Francisco, L., \& Benitti, F. B. V. (2014). Usability evaluation in Brazil: a systematic mapping. In Proceedings of the $9^{\text {th }}$ Iberian Conference on Information Systems and Technologies. Barcelona, Espanha.

Hammerschmidt, C. (2014). Tipografia em bulas de medicamento digitais para acesso ao Bulário Eletrônico em dispositivos de interação móvel. [Dissertação de mestrado]. Programa de Pós-graduação em Design, Universidade Federal do Paraná, Brasil.

Hornbaek, K., \& Oulasvirta, A. (2017). What is interaction? In Proceedings of the $2017 \mathrm{CHI}$ Conference on Human Factors in Computing Systems. Denver (CO): ACM Press.

ISO - International Organization for Standardization (2010). Ergonomics of human-system interaction - part 210: human-centred design for interactive systems.

Maguire, M. (2001). Methods to support human-centred design. Int. J. Human-Computer Studies, 55, $587-634$.

Moço, S. S. (1996). O uso de cenários como uma técnica de apoio para avaliações ergonômicas de softwares educativos. [Dissertação de mestrado]. Programa de Pósgraduação em Engenharia de Produção, Universidade Federal de Santa Catarina, Brasil.

Moran, K. (2019). Usability testing 101. In NN/g Nielsen Norman Group - articles. Disponível em: https://www.nngroup.com/articles/usability-testing-101/. Acesso em: 26.04.21.

Napo, P. (2015). Influências da interface gráfica em m-commerces sobre as motivações de compra online em smartphones. [Dissertação de mestrado]. Programa de Pós-graduação em Design, Universidade Federal do Paraná, Brasil.

Schumacher, R. M., \& Lowry, S. Z. (2010). Customized Common Industry Format Template for Electronic Health Record UsabilityTesting. NISTIR 7742. National Institute of Standards and Technology.

Rubin, J., \& Chisnell, D. (2011). Handbook of usability testing: how to plan, design and conduct effective tests. New Jersey: Wiley Publishing.

\section{Sobre os autores}

Stephania Padovani, Dra., UFPR, Brasil <stephania.padovani@gmail.com>

André Schlemmer, MSc., UFPR, Brasil <schlemmer.andre@gmail.com> 